MNF Interview with Dr. Yukihiko Hara, Mitsui Norin Co., Ltd.

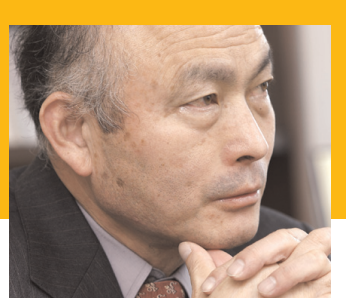

\section{Tea and Health}

Dr. Yukihiko Hara is one of the world's leading experts on the medicial benefits of green tea catechins, and has devoted 24 years of his life to research in this field. Dr. Hara has researched the medical benefits of catechins for a number of forms of cancer, Herpes virus, human papilloma virus, influenza virus, digestive disorders and other conditions. An inventor with over 120 patents describing the medicinal and other aspects of catechins, he has overseen the development of a large number of products that provide these benefits to the public. Dr. Hara is currently the Vice President of Mitsui Norin Co., Ltd., Tokyo, Japan.

\section{$M N F$ :}

You are known as one of the pioneers in promoting research on tea and health. How do you see the advances made in this area over the years?

\section{Hara:}

The advances made in research on the health of tea components, particularly catechins or tea polyphenols, are noteworthy. Against this background, consumers are becoming more aware of the health benefits of teas. The habit of drinking tea, green tea in particular, is gaining far more popularity than several years ago, and many people in the States seem to be consuming green tea as a daily beverage.

\section{$M N F$ :}

You are very creative in using tea and its products to improve the quality of people's daily lives in many different ways. Can you share some of the recent developments in this field?

\section{Hara:}

We have confirmed that tea catechins have a phenomenal effect in preventing the infectivity of the influenza virus, and this efficacy is practically commercialized as catechinimpregnated filters which are applied as mouth masks for flu-carriers or air cleaners for rooms or for automobiles.
Based on our basic patent, we are going to make the most of this property for the prevention of any flu virus pandemic in the coming years. The basic mechanism of this anti-flu property was confirmed as follows: tea catechins have a very strong affinity to such proteinous compounds as hemeagglutinin. Tea catechins bind firmly to hemeagglutinin at extremely low concentrations (thousandfold dilution of an ordinary cup) and that hinders the virus from entering into the human mucosal cells on catechin basis. Tea catechins are also known to reduce belly fat in humans if a good amount of green tea is drunk every day for months. This is assumed to be caused primarily by inhibiting the absorption of lipids in the intestine when tea catechins co-exist in the gut and in the liver by accelerating the catabolism of fat and inhibiting the reformation of triglycerides in the liver.

$M N F$ :

Japan is the major country in the world in the consumption of green tea. Do you feel a need to promote the habit of drinking green tea worldwide so more people can benefit from it? If so, how do you plan to do it?

\section{Hara:}

Yes, indeed. I feel strongly the need to promote the habit of drinking green tea worldwide. This doesn't mean, however, that the merit of black tea should be undervalued. Let me tell you why the study of green tea is far in advance of that of black tea, or oolong tea among others. Simply put, the chemistry of black tea is beyond current science while that of green tea is within our scope. Science demands consistency or repeatability of findings. Green tea allows these requirements chemically, while black tea chemistry is too diverse and complex to guarantee consistency or repeatability. This complexity derives from the manufacturing process of black tea. While the tea bush is growing in the tea garden, the major components of tea, i.e. tea catechins, accumulate in the shoots (in a few leaves at the tips) which are plucked and processed as tea. In the case of green tea, since the shoots are steamed (Japanese way) or panfried (Chinese way) soon after plucking, tea catechins remain in the tea as they are in the fresh leaves. In other words, tea catechins do not go through the process of enzymatic oxidation because of this heating process after plucking.

In comparison, in the process of manufacturing black tea, the plucked fresh tea leaves are rolled, crushed, torn and cut while the shoots are still alive. Such severe stress, while the cells are alive, causes various enzymatic reactions in the whole leaves. In this process, tea catechins are oxidized to form various dimeric, trimeric or oligomeric catechins which form the characteristic reddish color of black tea. We 
have isolated four kinds of natural catechins (whitish crystals) in tea leaves and characterized them chemically. Interestingly, the enzymatic activation process renders a very characteristic flavorsome aroma to black tea at the same time. Anyway, the problems of the chemistry of oligomeric catechins are enhanced by the stress to which tea leaves are exposed in each black tea factory.

$M N F$ :

Although there is substantial evidence from laboratory investigations showing the benefits of tea in warding off cancer, the results are mixed from epidemiological studies. How do you reconcile this disparity?

\section{Hara:}

This is a good question.

There are several reasons why the epidemiological studies do not necessarily reflect the anti-cancer effects shown in test tubes or in animal studies. Firstly, the teas drunk by consumers (subjects) are inconsistent. The tea itself as well as the way of brewing or the amount ingested vary from person to person. Secondly, these subjects are not homogeneous as compared to the laboratory animals. Thirdly, the amount of tea-soluble solids, consumed by the consumers in their ordinary lives, may be insufficient to prove the efficacy. In order to solve these problems, the US-NCI (National Cancer Institute, Dept. of Cancer Prevention) and we have been working for almost 10 years on the following strategy:

1) Definition of a high catechin powder of tea extract with good suppliability and high chemical consistency. This is called "Polyphenon E" which is composed of almost $90 \%$ tea catechins (more than $60 \%$ of epigallocatchin gallate (EGCg) and about $30 \%$ other catechins).

2) With this well-characterized composite, intensive and complete toxicity/safety studies have been conducted in cells, rodents, dogs and in humans. Thus, we know how much Polyphenon E is safe in human trials. In other words, we could increase the administration of Polyphenon E capsules within the confirmed safe dosage.

3) Recruitment of subjects who have specific pre-cancerous lesions (biomarkers). These subjects are apparently normal and have no diagnosable cancer but carry initial levels of DNA or physiological/histopathological pre-cancerous abnormalities. These subjects receive either Polyphenon E or placebo capsules in a randomized, double-blinded manner. After a certain period of administration, the progression or regression of the specific biomarkers is compared in the two groups.
In this way, chemopreventive studies are under way with the tea catechin composite, Polyphenon E, to confirm it as a chemopreventor in various pre-cancerous indications in several renowned cancer centers in the States. It will take another few years before definite results are available but the overall strategy warrants diverse clinical Phase 2 trials.

$M N F$ :

How do you see future directions for research on tea and human health?

Hara:

Tea catechins are considered to be effective in preventing various lifestyle-related, age-related diseases, i.e. so-called metabolic syndromes. Along with anti-cancer efficacy, studies on prevention of cardiovascular, cerebrovascular or diabetic diseases should be launched. Fat reduction is another large area to be tackled. Tea catechins have potent anti-microbial effects against such life-threatening infectious microbes as influenza viruses, the SARS virus, Vibrio cholerae, MRSA etc. Such anti-microbial potency should also be developed for practical uses. Along with these application studies, the fate of catechins in the intestine and the absorption into the body have to be studied in more detail (floral modulation, absorption, metabolism, distribution and excretion).

$M N F$ :

As a scientist working in an industrial setting, what do you think the role of the food industry should play in promoting and supporting research on tea and health?

Hara:

Wherever we are, we should do solid research and then promote evidence-based claims for the good health of consumers. Those in industry tend to be under pressure for commercial reasons but as long as we make a claim regarding human health, we should be strict about performing evidence-based studies. I assume that there is a tremendous possibility for most of the food industry to promote and benefit from "tea and health" by incorporating tea catechins (or other beneficial components in tea) in their products. There are various problems involved in those attempts, but they are worthwhile and warrant the challenge.

Interview by Fung-Lung Chung 